\title{
Cardiovascular Admissions, Readmissions, and Transitions of Care
}

\author{
Anna Marie Chang $\cdot$ Kristin L. Rising
}

Published online: 18 January 2014

(c) Springer Science+Business Media New York 2014

\begin{abstract}
Hospital 30-day readmissions have become a major priority for hospitals, which face penalties for excessive readmissions for acute myocardial infarction (AMI) and heart failure (HF). Thus, it is important for hospitals to understand the transitions of care that occur for both of these conditions, and what tools are available to guide the processes involved. A multi-disciplinary team including Emergency Medical Service providers, Emergency Medicine providers, cardiologists, hospitalists, pharmacists, nurses, case managers, and outpatient physicians can all be involved in the process of safely transitioning a patient between care settings. Small-scale studies in the geriatric population have shown improved transitions of care and decreased readmissions with these care teams. The emergency department is a key transition point for patients with AMI and HF, yet it is rarely identified and utilized as such in transitions of care interventions. Future research and implementation projects will need to refine and expand the role of the emergency department in the process.
\end{abstract}

Keywords Care coordination - Transitions of care . Acute myocardial infarction $\cdot$ Heart failure $\cdot$ Readmissions

\footnotetext{
A. M. Chang ( $₫)$

Department of Emergency Medicine, Oregon Health and Science University, MailCode CR 114, 3181 SW Sam Jackson Park Rd, Portland, OR 97239, USA

e-mail: changann@ohsu.edu

K. L. Rising

Department of Emergency Medicine, University of

Pennsylvania, 3400 Spruce Street, Philadelphia, PA 19104, USA
}

\section{Introduction}

Reduction of hospital readmissions has become a major priority for most hospitals, likely in large part due to the readmission penalty implemented by the Centers for Medicaid and Medicare Services (CMS) in October 2012. As a result of this legislation, hospitals are now penalized up to $1 \%$ of Medicare payments for excessive 30-day readmissions of patients with the primary diagnoses of acute myocardial infarction (AMI), heart failure (HF), and pneumonia [1•]. "Excessive readmissions" are determined by calculation of the excess readmission ratio, which is calculated separately for each condition, and is a comparison of each hospital's measured 30-day readmission rate for that condition to the national "expected" rate, based on an average hospital with similar patients. The penalty increased to $2 \%$ in October 2013, and is expected to increase again to $3 \%$ in October 2014. In addition to increasing the penalty rate, there are also discussions of increasing the number of conditions included in this program in future years [2]. It is estimated that 30-day mortality and readmission rates from 2005 to 2008 were 16.60 and $19.94 \%$ for AMI and 11.17 and $24.56 \%$ for HF, respectively [3•].

Though the emergency department (ED) has mostly been left out of initial interventions to reduce readmissions, this focus on preventing readmissions will inevitably have a trickle-down effect to the ED as it is the entry point for the majority of hospitalizations [4] and thus many potential readmissions. Recent work has found that patients who return to the ED after a prior inpatient discharge are readmitted about $50 \%$ of the time [5]. As hospitals develop means of proactively identifying patients who would qualify as a 30-day readmission case, EDs will likely be pushed to work with hospital case managers and discharge planners to 
determine if there are safe care settings for discharging these patients as an alternative to hospitalization.

This paper reviews data regarding care coordination and care transitions spanning from pre-hospital to post-discharge for acute MI and heart failure, highlighting ongoing coordination projects as well as potential strategies to be employed in future work.

\section{Definitions}

There are two terms used in this article that require clear definition: "transitions of care" and "care coordination". "Transitions of care" includes a broad range of time-limited services designed to ensure health care continuity, avoid preventable poor outcomes among at-risk populations, and promote the safe and timely transfer of patients from one level of care to another or from one type of setting to another [6•]. These transitions can occur at any health care exchange point, including from ED to inpatient status. "Care coordination", in contrast, is ideally an ongoing process defined as "the deliberate organization of patient care activities between two or more participants (including the patient) involved in a patient's care to facilitate the appropriate delivery of health care services". Organizing care involves the marshalling of personnel and other resources needed to carry out all required patient care activities and is often managed by the exchange of information among participants responsible for different aspects of care [7]. These concepts have become so widespread that there is a National Transitions of Care Coalition (http://www.ntocc.org) that has support guides and tools to assist health care professionals in executing effective transitions of care.

\section{Acute Myocardial Infarction and Transitions of Care}

In 2006, acute coronary syndrome (ACS) was the primary diagnosis for over 700,000 discharges [8•]. There are several transitions of care to be addressed and potentially improved within the realm of ACS, especially regarding management of ST-elevation myocardial infarction (STEMI).

\section{Emergency Medical Services to Hospital}

For patients who call 911 and activate Emergency Medical Services (EMS), direct care begins at the time at which the EMS provider arrives at the patient's side, and thus the initial transition of care is from the pre-hospital to hospital setting. The 2013 American College of Cardiology (ACC) and American Heart Association (AHA) Guidelines for the management of STEMI recommend a regionalized medical system to enable rapid recognition and timely reperfusion of patients with STEMI [9•]. According to the guidelines, EMS personnel should be accountable for obtaining a prehospital electrocardiogram (ECG), making the diagnosis of STEMI, activating the in-hospital response system, and deciding whether to transport the patient to a hospital that is capable of performing percutaneous coronary intervention (PCI). In addition, the guidelines suggest that "consideration should be given to the development of local protocols that allow preregistration and direct transport to the catheterization laboratory of a PCI-capable hospital (bypassing the ED) for patients who do not require emergent stabilization upon arrival" [9•].

Despite establishment of these guidelines, a recent study from the AHA Mission: Lifeline Program showed ED bypass occurring in only about $11 \%$ of STEMI cases. This poor adherence to these guidelines is likely a result of numerous factors, including multiple, separate EMS providers across the U.S. healthcare system with different individual protocols; ambulances without physician staffing; significant variation in geographic areas and terrain throughout the country; lack of a consistent information technology infrastructure to support the routine digital transmission of ECGs for physician review to minimize false catheterization laboratory activations; and dispersal of primary PCI services across a large number of hospitals [10].

An effective transition of care in the pre-hospital setting would include streamlined, standardized protocols for rapid transfer and transport of patients, as recommended by the AHA [11•]. These protocols would include standing orders for the care of STEMI patients with a well-defined care pathway, as well as a clearly defined plan for communicating vital information to in-hospital providers. For example, the D2B alliance (www.d2balliance.org) is an American College of Cardiology-sponsored initiative to achieve a door-to-balloon time of less than $90 \mathrm{~min}$ for at least $75 \%$ of non-transfer primary PCI patients. The DB2 alliance website includes such items as a STEMI alert checklist and a standard order set [12•]. These resources may be helpful for providers and hospitals to initiate their process of standardizing and streamlining the process for STEMI patients.

\section{Emergency Department to Hospital Admission}

Moving beyond the pre-hospital setting, EDs and hospitals can also use standardized protocols as guides in helping transition patients from the ambulance into the ED and subsequently to the cardiac catheterization laboratory, ensuring clear communication of care that has been provided and what is still required. 
In a time-sensitive and crucial condition such as ACS, it is important to clearly communicate between providers. Interviews with providers have identified numerous contributors to errors, from inaccurate to incomplete information, ED crowding and high workload, difficulty accessing information such as vital signs, and ambiguous responsibility for sign-out and follow-up [13]. It is crucial to have a way to deliver consistent, concise, and clear information, such as a checklist. Surgical safety checklists have been shown to decrease complication rates globally [14], and such checklists incorporated into the transfer of patients with ACS and STEMI may also improve the timeliness and quality of care delivered.

A number of tools have been developed from the inpatient side to encourage the initiation of as many lifesaving therapies as possible before patient discharge, including preprinted orders, care maps, discharge forms, physician/ nursing education plans, and treatment utilization reports [15]. These tools are designed to identify guideline-recommended treatments and encourage proper administration in appropriate patients. Often these tools help to prospectively identify major comorbidities and other factors that may be exacerbated as a result of a patient's MI and/or HF exacerbation, with the goal of being able to address these potential problems early in the process.

\section{Inpatient to Discharge}

Patient education and discharge planning as well as timely follow-up and outpatient monitoring have significant room for improvement. The Society of Hospital Medicine has established core competencies for hospitalists who manage patients with ACS [16॰]. According to these competencies, discharge planning and care coordination should begin as soon as the patient is admitted to the hospital. Regarding the discharge process itself, the ACC/AHA guidelines recommend that prior to hospital discharge, patients and caregivers should be provided with well-understood instructions for medications and follow-up appointments. In addition, they should all be provided with instructions on behavioral changes such as smoking cessation, weight management and exercise, and blood pressure, cholesterol, and diabetes control [9•, 17•, 18]. In addition to acute rehabilitation, many patients may also benefit from coordination of rehabilitation for secondary prevention [19•]. Despite these guidelines for in-hospital and dischargespecific care, almost one-third of patients in one study reported receiving less than 1 day's advance notice of discharge, and although one-third of patients were discharged with a scheduled appointment, less than half could accurately recall details of the appointment [13]. In addition, cardiac rehabilitation programs remain underutilized across the United States, with an estimated participation rate of only $10-20 \%$ of eligible patients per year who experience an acute MI [19•].

\section{Congestive Heart Failure and Transitions of Care}

An estimated 5.1 million Americans 20 years and older have $\mathrm{HF}$, which is projected to increase $25 \%$ by 2030 [8•]. There are over $650,000 \mathrm{ED}$ visits per year for acute HF exacerbations, and about $80 \%$ of these visits result in admission to the hospital [20•]. In addition, $25 \%$ of hospital discharges for HF will result in a 30-day readmission, a rate which has been relatively stable in past years $[21 \bullet$, $22 \cdot, 23 \bullet]$.

\section{EMS to Hospital}

The ACC/AHA and European College of Cardiology have jointly established guidelines for the management of acute HF patients. These guidelines do not, however, contain specific recommendations on early management. Thus, a multi-national group of experts convened in 2006 specifically to develop recommendations for early management of acute HF exacerbations, acknowledging "all acute heart failure syndrome patients should have appropriate goaldirected treatment started as early as possible." Pre-hospital recommendations included rapid establishment of the diagnosis, transfer as quickly as possible to a hospital (preferably with cardiology service and cardiac care unit [CCU]), establishment of communication between EMS personnel and the receiving hospital, and consideration of use of continuous positive airway pressure (CPAP) [24].

\section{Emergency Department to Hospital Admission}

The ED is the primary point of admission for patients with HF exacerbations, and should be a pivotal player in discussions regarding improving care transitions and reducing subsequent recurrent care utilization. In addition, as placement in the observation unit (OU) does not count as an admission (or a readmission), providers are likely to receive increasing pressure to place patients in the OU as opposed to inpatient whenever possible. At this time, however, there are limited studies assessing ED transitions for HF patients, largely because the vast majority of patients are admitted to the hospital and the focus of studies has been on transitions home from the inpatient setting. Currently four out of every five patients presenting to the ED with $\mathrm{HF}$ are admitted to the hospital, though studies have suggested that up to $50 \%$ of patients may be safe for a shorter observation stay or even for discharge home [20•, 25]. One study raises "the need for skillful coordination of the transition of care" as a "significant hurdle" in placing patients in the observation 
unit or discharging them home from the ED instead of admitting them to the hospital [25]. Increased efforts on establishing clear communication pathways with outpatient cardiologists and a mechanism by which patients can be scheduled for an outpatient follow-up visit within the following 1-2 days may assist ED providers in safely discharging a set of HF patients home [20•].

\section{Inpatient to Discharge}

The time of transition home from the hospital, referred to by some as the "vulnerable phase" [26•], has received significant focus in efforts for reducing readmissions for $\mathrm{HF}$, in large part because of the significant amount of care coordination that needs to take place. This coordination ideally involves detailed communication between multiple sources, including the patient and family, inpatient and outpatient providers, pharmacists, and other case managers, and has historically varied widely between hospitals.

There have been a number of studies of innovative approaches to reducing 30-day readmission rates for $\mathrm{HF}$ that have addressed different aspects of the discharge process and have shown improved outcomes including reduced 30-day readmissions and/or improvement in other measures of morbidity and mortality. While the details of these interventions vary, they are generally aimed at addressing one or more of the following categories: improved discharge instructions [27•], patient empowerment/motivation [28], comprehensive medication reconciliation [27•], assistance with linkage to short-term outpatient follow-up providers [29], and the provision of various home health services [30].

The Joint Commission on Accreditation of Healthcare Organizations (JCAHO) has established 3 performance measures specific to the discharge of patients with $\mathrm{HF}$, one of which mandates the administration of discharge instructions to all patients with $\mathrm{HF}$, as follows (HF-1): "Heart failure patients discharged home with written instructions or educational materials given to patient or caregiver at discharge or during the hospital stay addressing all of the following: activity level, diet, discharge medications, follow-up appointment, weight monitoring, and what to do if symptoms worsen" [31]. Despite having identified hospital discharge as a critical time for care coordination, recent studies have identified many areas of shortfall in communication and post-discharge planning.

One study of early outpatient follow-up found that only $38 \%$ of patients had follow-up within 7 days of discharge [32]. Bradley et al. [21•] performed a comprehensive review of multiple components of the discharge processes for patients with HF or AMI. They received response from 537 hospitals, $87 \%$ of which reported having a QI team devoted to reducing preventable admissions for patient with $\mathrm{CHF}$, and found that close to $50 \%$ of hospitals discharged CHF patients without a plan for managing changes in their condition, and fewer than half gave patients who received home health services an inpatient point of contact for questions. In addition, less than $55 \%$ of hospitals "usually" or "always" discharged patients with an outpatient follow-up appointment, only $37 \%$ alerted the outpatient provider of a patient discharge within $48 \mathrm{~h}$, and less than $65 \%$ sent a discharge summary to outpatient providers "most" or "all" of the time. Only $20 \%$ of hospitals gave patients a discharge summary, and less than $70 \%$ had a discharge summary ready for viewing within 7 days after discharge. In cases of discharge to a skilled nursing facility, $61 \%$ ensured nurse-to-nurse report prior to transfer and only $47 \%$ always provided a discharge summary.

There is a multi-rung ladder of responsibility at the time of discharge home. Patients and families must have a clear understanding of the disease process, including early signs of decompensation such as subtle weight increases. Inpatient providers must provide timely and complete discharge information to the outpatient providers as well as assisting in setting up a follow-up appointment for the patient shortly after discharge. Outpatient providers, in return, must put processes in place to allow for timely scheduling of patients both after discharge and for urgent appointment as needed.

The OPTIMIZE-HF program was developed to help hospitals improve their systems for treating HF patients, and includes provision of a number of standardized pathways and checklists for hospitals. Studies of hospitals enrolled in this program have shown increases in adherence to all 4 JCAHO measures of quality in HF patients, thought likely attributable both to improved measurement of performance and availability of standardized tools [33]. Thus, a reasonable step forward in ensuring best practices across the nation may be universal implementation of these checklists to facilitate streamlined and comprehensive discharge of HF patients. Once home, patients may also benefit from improved mechanisms for receiving remote care and monitoring. A recent review of structured telephone support and telemonitoring as a means of caring for HF patients at home found that both methods were effective in reducing the overall risk of all-cause mortality and HF-related hospitalizations, as well as in improving general quality of life and reducing healthcare costs. Considering these findings, there may be an increasing role for telehealth in facilitating continued care coordination and monitoring at home.

\section{Do Transitional Interventions Work?}

In a recent meta-analysis of interventions to decrease 30-day readmissions, the authors created a taxonomy of the interventions, including pre-discharge interventions, post- 
discharge interventions, and interventions bridging the transitional gap [34•]. Overall, there were 12 categories of interventions that were evaluated, and there was significant variation in the components of the bundles. Only 16 of the 43 studies utilized a randomized controlled trial design.

Several key projects and initiatives have focused on patient transitions to the outpatient setting, a few of which are highlighted here. Project RED (Re-Engineered Discharge) was designed by researchers at Boston University Medical Center with funding from the Agency for Healthcare Research and Quality [35•]. The intervention includes a toolkit to help train staff on 12 discrete components of the discharge process, including items such as planning for follow-up visits, reconciling medications, and reviewing discharge planning with patients. Results of a randomized controlled trial showed that those patients in the intervention group had lower rates of hospital utilization than those in the usual care group [35॰]. Project BOOST (Better Outcomes by Optimizing Safe Transitions), sponsored in part by the Society for Hospital Medicine, identifies general medicine high-risk patients at the time of admission with the goal of reducing 30-day readmission rates [36 $]$. This program helps identify patients at risk of readmission early, and assists the site in developing tools to optimize discharge process. The teach-back process is utilized to ensure patients understand care plans, self-care instructions, and follow-up appointments. Preliminary data from sites that implemented Project BOOST had a reduction in their 30-day readmission rates from 14.7 to $12.7 \%$ at 12 months after implementation [36 ]. The Care Transitions Intervention was developed by a team led by Eric Coleman in Colorado, and decreased rehospitalization rates at both 30 and 90 days [3740]. This intervention focuses on the utilization of a "transitions coach" to encourage the patient and family to take a more active role in his health. Finally, Naylor et al. [6•, 41, 42] developed a nurse-led transitional care model that was found to decrease readmissions specifically in the elderly population.

These relatively small-scale studies have shown the efficacy of transitional care tools, with the next step that these tools need to be further disseminated and implemented in a broad population. In addition, it may be helpful to further test the tools for specific disease entities such as acute coronary syndrome and heart failure. Cardiovascular patient care requires a multidisciplinary approach. Decreasing readmissions is only one part of the pathway.

\section{Compliance with Ethics Guidelines}

Conflict of Interest This paper was supported in part by grants from NHLBI to Anna Marie Chang. Kristin Rising declares that she has no conflicts of interest.

Human and Animal Rights and Informed Consent This article does not contain any studies with human or animal subjects performed by any of the authors.

\section{References}

Papers of particular interest, published recently, have been highlighted as:

- Of importance

1. - Readmissions Reduction Program-Centers for Medicare and Medicaid Services. 2012. http://www.cms.gov/Medicare/MedicareFee-for-Service-Payment/AcuteInpatientPPS/ReadmissionsReduction-Program.html. The official website of the Center for Medicare \& Medicaid Services for the Readmissions Reduction Program, offering an overview of the program, how readmission rates are calculated, and file links for supplemental information.

2. Rao J. Armed with bigger fines, medicare to punish 2,225 hospitals for excess readmissions-Kaiser Health News. 2013. http://www. kaiserhealthnews.org/Stories/2013/August/02/readmissionpenalties-medicare-hospitals-year-two.aspx. Accessed 21 Aug 2013.

3. - Krumholz HM, Lin Z, Keenan PS, et al. Relationship between hospital readmission and mortality rates for patients hospitalized with acute myocardial infarction, heart failure, or pneumonia. JAMA. 2013;309(6):587-93. doi:10.1001/jama.2013.333. A large-scale analysis of Medicare data assessing the association between risk-standardized mortality and readmission rates for patients hospitalized with acute myocardial infarction, pneumonia, and heart failure.

4. Kocher KE, Dimick JB, Nallamothu BK. Changes in the source of unscheduled hospitalizations in the United States. Med Care. 2013;51(8):689-98. doi:10.1097/MLR.0b013e3182992c7b.

5. Rising KL, White LF, Fernandez WG, Boutwell AE. Emergency department visits after hospital discharge: a missing part of the equation. Ann Emerg Med. 2013;62:145-50.

6. - Naylor MD, Aiken LH, Kurtzman ET, Olds DM, Hirschman KB. The Importance of Transitional Care in Achieving Health Reform. Health Aff (Millwood). 2011;30(4):746-54. doi:10.1377/ hlthaff.2011.0041. This article highlights the definitions and differences between transitions of care and care coordination and presents recent literature and reviews on these topics.

7. Chapter 2. What is care coordination? From care coordination measures atlas. Agency for Healthcare Research and Quality, Rockville, MD. 2011. http://www.ahrq.gov/professionals/systems/ long-term-care/resources/coordination/atlas/index.html. Accessed Jan 2011

8. - Go AS, Mozaffarian D, Roger VL, et al. Heart disease and stroke statistics-2013 update: a report from the American Heart Association. Circulation. 2013;127(1):e6-245. doi:10.1161/CIR. 0b013e31828124ad. The 2013 update of an annual report published by the American Heart Association in conjunction with the Centers for Disease Control and Prevention, the National Institutes of Health, and other government agencies to provide up-to-date statistics on heart disease, stroke, and other vascular conditions.

9. • O'Gara PT, Kushner FG, Ascheim DD, et al. 2013 ACCF/AHA guideline for the management of ST-elevation myocardial infarction: a report of the American College of Cardiology Foundation/American Heart Association task force on practice guidelines. Circulation. 2013;127(4):e362-425. doi:10.1161/CIR. 0b013e3182742cf6. Report of the most recent guidelines from the ACCFA/AHA regarding care of STEMI patients. Includes the summary of an extensive literature review conducted from November 2010 through August 2012, with over 650 references included in the report.

10. Bagai A, Jollis JG, Dauerman HL, et al. Emergency department bypass for ST-segment-elevation myocardial infarction patients identified with a prehospital electrocardiogram a report from the 
American Heart Association mission: lifeline program. Circulation. 2013;128(4):352-9. doi:10.1161/CIRCULATIONAHA.113. 002339.

11. American Heart Association: Recommendations for criteria for STEMI systems of care. 2013. http://www.heart.org/HEARTORG/ HealthcareResearch/MissionLifelineHomePage/EMS/Recommen dations-for-Criteria-for-STEMI-Systems-of-Care_UCM_312070_ Article.jsp. Accessed 28 Aug, 2013. This webpage provided AHA guidelines for providers and administrators on developing appropriate STEMI systems of care.

12. - D2B Implementation Manual: American College of Cardiology. 2013. http://www.d2balliance.org/D2BImplementationManual/ tabid/141/Default.aspx. Accessed 28 Aug, 2013. A manual developed by the American College of Cardiology to assist programs in reducing door-to-balloon times for patients requiring PCI intervention.

13. Horwitz LI, Moriarty JP, Chen C, et al. Quality of discharge practices and patient understanding at an academic medical center. JAMA Intern Med. 2013. doi:10.1001/jamainternmed. 2013.9318.

14. Weiser TG, Haynes AB, Dziekan G, et al. Effect of a 19-item surgical safety checklist during urgent operations in a global patient population. Ann Surg. 2010;251(5):976-80. doi:10.1097/ SLA.0b013e3181d970e3.

15. Peterson ED, Albert NM, Amin A, Patterson JH, Fonarow GC. Implementing critical pathways and a multidisciplinary team approach to cardiovascular disease management. Am J Cardiol. 2008;102(Suppl):47G-56G. doi:10.1016/j.amjcard.2008.06.011.

16. - Villanueva T. Transitioning the patient with acute coronary syndrome from inpatient to primary care. J Hosp Med. 2010;5:S8-14. doi:10.1002/jhm.829. This article discusses key issues with the transitions of care from hospital to home setting for acute coronary syndromes. A useful guide for ED physicians, hospitalists, and outpatient providers.

17. - Anderson JL, Adams CD, Antman EM, et al. 2012 ACCF/AHA focused update incorporated into the ACCF/AHA 2007 guidelines for the management of patients with unstable angina/nonST-elevation myocardial infarction a report of the American College of Cardiology Foundation/American Heart Association Tas. Circulation. 2013;127(23):e663-828. doi:10.1161/CIR. 0b013e31828478ac. The most recent report from the ACCF/ AHA regarding management of patients with unstable angina and non-STEMI, including comprehensive recommendations and a total of 1113 references.

18. Ades PA. Cardiac rehabilitation and secondary prevention of coronary heart disease. N Engl J Med. 2001;345(12):892-902. doi:10.1056/NEJMra001529.

19. - Leon AS, Franklin BA, Costa F, et al. Cardiac Rehabilitation and secondary prevention of coronary heart disease: an American Heart Association scientific statement from the Council on Clinical Cardiology (subcommittee on exercise, cardiac rehabilitation, and prevention) and the Council on Nutrition, Physical Activity, and Metabolism (Subcommittee on Physical Activity), in collaboration with the American Association of Cardiovascular and Pulmonary Rehabilitation. Circulation. 2005;111(3):369-76. doi:10.1161/01.CIR.0000151788.08740.5C. An update to the 2005 AHA statement report on cardiac rehabilitation, providing a review of recommended components of cardiac rehabilitation and secondary prevention.

20. - Weintraub NL, Collins SP, Pang PS, et al. Acute heart failure syndromes: emergency department presentation, treatment, and disposition: current approaches and future aims: a scientific statement from the American Heart Association. Circulation. 2010;122(19):1975-96. doi:10.1161/CIR.0b013e3181f9a223. A scientific statement from the AHA regarding ED management of heart failure, including recommendations for next studies and potential areas of care improvement.

21. - Bradley EH, Curry L, Horwitz LI, et al. Contemporary evidence about hospital strategies for reducing 30-day readmissions a national study. J Am Coll Cardiol. 2012;60(7):607-14. doi:10. 1016/j.jacc.2012.03.067. Results of a web-based survey to a national cross-section of hospitals enrolled in the Hospital to Home quality improvement initiative to determine strategies employed by hospitals for reducing 30-day readmissions for patients with $\mathrm{HF}$ or AMI.

22. - Ross JS, Chen J, Lin ZQ, et al. Recent national trends in readmission rates after heart failure hospitalization. Circ Heart Fail. 2010;3(1):97-103. doi:10.1161/CIRCHEARTFAILURE. 109.885210.Recent. A national study using Medicare data to assess recent changes in 30-day readmission rates for $\mathrm{HF}$, finding no significant change from 2004 to 2006.

23. - Bernheim SM, Grady JN, Lin Z, et al. National patterns of riskstandardized mortality and readmission for acute myocardial infarction and heart failure. Update on publicly reported outcomes measures based on the 2010 release. Circ Cardiovasc Qual Outcomes. 2010;3(5):459-67. doi:10.1161/CIRCOUTCOMES. 110.957613. A national study using Medicare data to assess hospital performance in 2010 on measures of mortality and readmission rates for patients with $A M I$ and $H F$.

24. Mebazaa A, Gheorghiade M, Piña IL, et al. Practical recommendations for prehospital and early in-hospital management of patients presenting with acute heart failure syndromes. Crit Care Med. 2008;36(1 Suppl):S129-39. doi:10.1097/01.CCM.0000296 274.51933.4C

25. Collins SP, Pang PS, Fonarow GC, Yancy CW, Bonow RO, Gheorghiade M. Is hospital admission for heart failure really necessary? The role of the emergency department and observation unit in preventing hospitalization and rehospitalization. JACC. 2013;61(2):121-6. doi:10.1016/j.jacc.2012.08.1022.

26. - Gheorghiade M, Vaduganathan M, Fonarow GC, Bonow RO. Rehospitalization for heart failure: problems and perspectives. J Am Coll Cardiol. 2013;61(4):391-403. doi:10.1016/j.jacc.2012. 09.038. A useful overview of potential approaches to reducing readmissions and improving care coordination for patients with $H F$.

27. - Jovicic A, Holroyd-Leduc JM, Straus SE. Effects of self-management intervention on health outcomes of patients with heart failure: a systematic review of randomized controlled trials. BMC Cardiovasc Disord. 2006;6:43. doi:10.1186/1471-2261-6-43. A useful review summarizing the randomized control trials that have been performed to assess efficacy of self-management interventions on HF outcomes.

28. Austin LS, Landis CO, Hanger KH. Extending the continuum of care in congestive heart failure: an interactive technology selfmanagement solution. J Nurs Adm. 2012;42(9):442-6. doi:10. 1097/NNA.0b013e3182668342.

29. Hernandez AF, Greiner MA, Fonarow GC, et al. Relationship between early physician follow-up and 30-day readmission among medicare beneficiaries hospitalized for heart failure. JAMA. 2010;303(17):1716-22.

30. Delgado-Passler P, McCaffrey R. The influences of postdischarge management by nurse practitioners on hospital readmission for heart failure. J Am Acad Nurse Pract. 2006;18(4):154-60. doi:10. 1111/j.1745-7599.2006.00113.x.

31. The Joint Commission. Specifications manual for National Hospital inpatient quality measures-heart failure measure set; HF-1. http://www.jointcommission.org/core_measure_sets.aspx.

32. Kociol RD, Greiner MA, Fonarow GC, et al. Associations of patient demographic characteristics and regional physician density with early physician follow-up among medicare beneficiaries 
hospitalized with heart failure. Am J Cardiol. 2011;108(7): 985-91. doi:10.1016/j.amjcard.2011.05.032.

33. Fonarow GC, Abraham WT, Albert NM, et al. Influence of a performance-improvement initiative on quality of care for patients hospitalized with heart failure. Arch Intern Med. 2007; 167(14):1493-502.

34. - Hansen LO, Young RS, Hinami K, Leung A, Williams MV. Interventions to reduce 30-day rehospitalization: a systematic review. Ann Intern Med. 2011;155(8):520-8. doi:10.1059/00034819-155-8-201110180-00008. An important review and summary of interventions that have been developed to reduce 30-day hospital readmissions rates for all conditions, including a total of 43 articles describing the various interventions.

35. - Jack BW, Chetty VK, Anthony D, et al. A reengineered hospital discharge program to decrease rehospitalization: a randomized trial. Ann Intern Med. 2009;150(3):178-87. Primary results of implementation of Project RED at a single center. Includes basic details of the components of the intervention.

36. - Hansen LO, Greenwald JL, Budnitz T, et al. Project BOOST: effectiveness of a multihospital effort to reduce rehospitalization. J Hosp Med. 2013;8(8):421-7. doi:10.1002/jhm.2054. Initial results of Project BOOST, which providers and administrators can use to obtain more information and guidelines for developing a readmissions program.

37. Coleman EAPC. The care transitions intervention: results of a randomized controlled trial. Arch Intern Med. 2006;166(17): 1822-8. doi:10.1001/archinte.166.17.1822.

38. Coleman EA, Smith JD, Raha D, Min S. Posthospital medication discrepancies: prevalence and contributing factors. Arch Intern Med. 2005;165(16):1842-7. doi:10.1001/archinte.165.16.1842.

39. Misky GJ, Wald HL, Coleman EA. Post-hospitalization transitions: examining the effects of timing of primary care provider follow-up. J Hosp Med. 2010;5(7):392-7. doi:10.1002/jhm.666.

40. Coleman EA, Smith JD, Frank JC, Eilertsen TB, Thiare JN, Kramer AM. Development and testing of a measure designed to assess the quality of care transitions. Int J Integr Care. 2002; 2:e02.

41. Naylor MD, Brooten D, Campbell R, et al. Comprehensive discharge planning and home follow-up of hospitalized elders: a randomized clinical trial. JAMA. 1999;281(7):613-20.

42. Naylor MD, Brooten DA, Campbell RL, Maislin G, McCauley KM, Schwartz JS. Transitional care of older adults hospitalized with heart failure: a randomized, controlled trial. J Am Geriatr Soc. 2004;52(5):675-84. doi:10.1111/j.1532-5415.2004.52202.x. 